\title{
DERIVATIVES OF INFINITE ORDER
}

\section{R. P. BOAS, JR. AND K. CHANDRASEKHARAN}

Let $f(x)$ have derivatives of all orders in $(a, b)$. If, as $n \rightarrow \infty, f^{(n)}(x)$ $\rightarrow g(x)$ uniformly, or even boundedly, dominatedly or in the mean, then $g(x)$ is necessarily of the form $k e^{x}$, where $k$ is a constant; in fact, if $c \in(a, b)$,

$$
f^{(n-1)}(x)-f^{(n-1)}(c) \rightarrow \int_{e}^{x} g(t) d t
$$

and so

$$
g(x)-g(c)=\int_{0}^{x} g(t) d t .
$$

It then follows first that $g(x)$ is continuous, then that $g(x)$ is differentiable in $(a, b)$, finally that $g^{\prime}(x)=g(x)$ and so $g(x)=a e^{x}$.

If $f^{(n)}(x)$ approaches a limit only for one value of $x$, however, it does not necessarily do so for other values of $x$. On the other hand, G. Vitali [10 $]^{1}$ and V. Ganapathy Iyer [6] showed that if $f(x)$ is analytic in $(a, b)$ and $f^{(n)}(x)$ approaches a limit for one $x_{0} \in(a, b)$, then $f^{(n)}(x)$ converges uniformly in each closed subinterval of $(a, b)$. Ganapathy Iyer asked two questions in this connection:

(I) If $f^{(n)}(x) \rightarrow g(x)$ for each $x$ in $(a, b)$, where $g(x)$ is finite, does $g(x)=k e^{x}$ ?

(II) If $f(x)$ belongs to a quasianalytic class in $(a, b)$ and $\lim _{n \rightarrow \infty} f^{(n)}\left(x_{0}\right)$ exists for a single $x_{0}$, does $\lim _{n \rightarrow \infty} f^{(n)}(x)$ exist for every $x$ in $(a, b)$ ?

We shall show that the answer to both questions is yes. We also indicate some possible generalizations.

We first answer (I).

Theorem 1. If $f^{(n)}(x) \rightarrow g(x)$ for each $x$ in $(a, b)$, where $g(x)$ is finite, then $f(x)$ is analytic in $(a, b)$.

It follows from Ganapathy Iyer's result that then $g(x)=k e^{x}$.

PRoof. At each point $x$ of $(a, b)$ form the Taylor series of $f(x)$. The radius of convergence of this series, as a function of $x$, has a positive

Presented to the Society, September 5, 1947; received by the editors May 29, 1947.

${ }^{1}$ Numbers in brackets refer to the references cited at the end of the paper. 
lower bound; in fact, it is infinite for each $x$. By a known theorem $[2,5,11]$ (stated with an incomplete proof by Pringsheim $[9, \mathrm{p}$. $180]), f(x)$ is analytic in $(a, b)$.

Next we answer (II).

Theorem 2. If $f(x)$ belongs to a Denjoy-Carleman quasianalytic class in the (open) interval $(a, b)$, and if $f^{(n)}\left(x_{0}\right) \rightarrow L$ for one $x_{0}$ in $(a, b)$, then $f(x)$ is analytic in $(a, b)$.

Again, by the result of Vitali and Ganapathy Iyer it follows that $f^{(n)}(x) \rightarrow L e^{x-x_{0}}$ in $(a, b)$.

PROoF. We say that $f(x) \in C\left\{M_{n}\right\}$ if $\left|f^{(n)}(x)\right| \leqq k^{n} M_{n}, x \in I$, for each closed subinterval $I$ of $(a, b)$, where $k$ depends on $f(x)$ and on $I$. The class $C\left\{M_{n}\right\}$ is quasianalytic if $\sum M_{n}^{-1 / n}<\infty$; in this case any two functions of the class which coincide, together with all their derivatives, at $x_{0} \in(a, b)$, are identical. It is known $[3,8]$ that $C\left\{M_{n}\right\}$ is identical with the class $C\left\{M_{n}^{\circ}\right\}$ obtained by a certain regularizing process; the only property of $M_{n}^{0}$ which we need here is that $M_{n+1}^{o} / M_{n}^{o}$ is nondecreasing. It follows that every class $C\left\{M_{n}\right\}$, except the trival class $C\{0\}$, contains $C\{1\}$. This seems to have been first pointed out explicitly by T. Bang $[1$, p. 16]; we reproduce the simple proof.

We have to show that $k_{1}^{n} \leqq k_{2}^{n} M_{n}$, or that $M_{n}^{o} \geqq k_{3}^{n}$ for some $k_{8}$. Now we have $M_{n}^{o} / M_{n-1}^{o} \geqq M_{1}^{o} / M_{0}^{o}=\alpha$, say. Hence $M_{n}^{o} \geqq M_{n-1}^{o} \alpha \geqq M_{n-2}^{o} \alpha^{2}$ $\geqq \cdots \geqq \alpha^{n} M_{0}^{o}$.

Now suppose that $f^{(n)}\left(x_{0}\right) \rightarrow L$ and let

$$
g(x)=\sum_{k=0}^{\infty} f^{(k)}\left(x_{0}\right)\left(x-x_{0}\right)^{k} / k !
$$

For some number $Q,\left|f^{(n)}\left(x_{0}\right)\right| \leqq Q$. Hence

$$
\left|g^{(n)}(x)\right|=\left|\sum_{k=0}^{\infty} f^{(n+k)}\left(x_{0}\right)\left(x-x_{0}\right)^{k} / k !\right| \leqq Q e^{x-x_{0}}
$$

and so $g(x) \in C\{1\}$; hence $g(x) \in C\left\{M_{n}\right\}$. But $g^{(n)}\left(x_{0}\right)=f^{(n)}\left(x_{0}\right)$ for every $n$ and so $f(x) \equiv g(x)$, an analytic function.

A natural generalization of the problem is to interpret the relation $f^{(n)}(x) \rightarrow g(x)$ in a generalized sense. For example, if $f^{(n)}(x) \rightarrow g(x)(C, 1)$, dominatedly, the proof given in $\$ 1$ shows that $g(x)=k e^{x}$; this proof, in fact, applies to any generalized limit such that $s_{n-1}(x)$ converges to the same limit as $s_{n}(x)$ (see [4, p. 418], [7] for discussions of such generalized limits, which include, in particular, $(C, k), k>-1)$.

We can also replace $f^{(n)}(x) \rightarrow g(x)$ by $f^{(n)}(x) / \lambda_{n} \rightarrow g(x),\left\{\lambda_{n}\right\}$ a given se- 
quence of constants. We give two simple theorems in this direction.

THEOREM 3. Let

$$
\lim _{n \rightarrow \infty} f^{(n)}(x) / \lambda_{n}=g(x), \quad a \leqq x \leqq b .
$$

(i) If $\lim \inf \left|\lambda_{n-1} / \lambda_{n}\right|=0$ and (1) holds uniformly, $g(x) \equiv 0$ in $a \leqq x \leqq b$.

(ii) If lim inf $\left|\lambda_{n-1} / \lambda_{n}\right|>0$ and (1) holds dominatedly, $g(x)=k e^{b x}$.

The example $f(x)=1 / x, \lambda_{n}=(-1)^{n} n !, a=1, b=2$ shows that uniformity is essential in (i). It would be interesting to know whether (without uniformity) there can be an exceptional point in the interior of $(a, b)$; if $f(x)$ is analytic, there cannot, as the next theorem shows.

THEOREM 4. If

$$
\limsup _{n \rightarrow \infty} n^{-1}\left|\lambda_{n}\right|^{1 / n}<\infty
$$

and (1) is true for each $x$ in $a<x<b$, then $g(x)=k e^{b x}$ in $a<x<b$.

If $f(x)$ is analytic, $\lim \sup n^{-1}\left|f^{(n)}(x)\right|^{1 / n}<\infty$ for each $x$ and hence either (2) is true or (1) implies $g(x) \equiv 0$.

Proof of TheOREM 3. We observe that if $a<c<b$

$$
\lim _{n \rightarrow \infty} \frac{\lambda_{n-1}}{\lambda_{n}}\left\{\frac{f^{(n-1)}(x)}{\lambda_{n-1}}-\frac{f^{(n-1)}(c)}{\lambda_{n-1}}\right\}=\int_{0}^{x} g(t) d t .
$$

If (i) of Theorem 3 is true, the left side of (3) approaches zero as $n \rightarrow \infty$ through a suitable sequence; hence $g(x)=0$ almost everywhere; but $g(x)$ is continuous because (1) holds uniformly, and so $g(x) \equiv 0$.

If (ii) is true and $\lim \left|\lambda_{n-1} / \lambda_{n}\right|=\infty, \lambda_{n} \rightarrow 0$ and so $f^{(n)}(x) \rightarrow 0$; otherwise, for some sequence of $n$ 's, $\lambda_{n-1} / \lambda_{n} \rightarrow L$, where $L \neq 0, L \neq \infty$. Then (3) gives

$$
L\{g(x)-g(c)\}=\int_{0}^{x} g(t) d t
$$

and hence $g(x)=k e^{x / L}$.

Proof of Theorem 4. We have from (1), for each $x$ and for $n>n_{x},\left|f^{(n)}(x)\right| \leqq(1+g(x))\left|\lambda_{n}\right|$, and so

$$
\limsup _{n \rightarrow \infty}\left|f^{(n)}(x) / n !\right|^{1 / n} \leqq \limsup _{n \rightarrow \infty}\left|\lambda_{n}\right|^{1 / n} /(n / e)<\infty \text {. }
$$

The reasoning given for Theorem 1 now applies. 


\section{REFERENCES}

1. T. Bang, Om quasi-analytiske Funktioner, Copenhagen thesis, 1946.

2. R. P. Boas, Jr., A theorem on analytic functions of a real variable, Bull. Amer. Math. Soc. vol. 41 (1935) pp. 233-236.

3. H. Cartan and S. Mandelbrojt, Solution du probleme d'equivalence des classes de fonctions indefiniment dérivables, Acta Math. vol. 72 (1940) pp. 31-49.

4. P. Dienes, The Taylor series, Oxford, 1931.

5. V. Ganapathy Iyer, Sur un probleme de M. Carleman, C. R. Acad. Sci. Paris. vol. 199 (1934) pp. 1371-1373.

6. - On singular functions, J. Indian Math. Soc. N.S. vol. 8 (1944) pp. 94108.

7. J. D. Hill, Some properties of summability, Duke Math. J. vol. 9 (1942) pp. 373381.

8. S. Mandelbrojt, Analytic functions and classes of infinitely differentiable functions, Rice Institute Pamphlet vol. 29 (1942) pp. 1-142.

9. A. Pringsheim, Zur Theorie der Taylor'schen Reihe und der analytischen Funktionen mit beschränkten Existenzbereich, Math. Ann. vol. 42 (1893) pp. 153-184.

10. G. Vitali, Sui limiti per $n=\infty$ delle derivate $n^{\text {ma }}$ delle funzioni analitiche, Rend. Circ. Mat. Palermo vol. 14 (1900) pp. 209-216.

11. Z. Zahorski, Sur l'ensemble des points singuliers d'une fonction d'une variable reelle admettant les dérivees de tous les ordres, Fund. Math. vol. 34 (1947) pp. 183-245.

BROWN UNIVERSITY AND

InSTITUte gor AdVANCEd StUdy 